\title{
Composición florística y evaluación de la degradación del bosque pantanoso costero de temu-pitra en la Región de La Araucanía, Chile
}

\section{Floristic composition and evaluation of the degradation of the swampy coastal forest of temu-pitra in the Araucanía Region, Chile}

\author{
Enrique Hauenstein ${ }^{1 *}$, Fernando Peña-Cortés ${ }^{1}$, Carlos Bertrán ${ }^{2}$, Jaime Tapia ${ }^{3}$, luis Vargas-

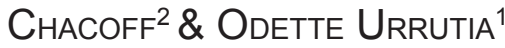

${ }^{1}$ Escuela de Ciencias Ambientales, Facultad de Recursos Naturales, Universidad Católica de Temuco. Casilla 15-D, Temuco, Chile.

${ }^{2}$ Instituto de Ciencias Marinas y Limnológicas, Facultad de Ciencias, Universidad Austral de Chile. Casilla 567, Valdivia, Chile. ${ }^{3}$ Instituto de Química de Recursos Naturales, Universidad de Talca. Casilla 747, Talca, Chile.

*ehauen@uct.cl

\begin{abstract}
RESUMEN
El área costera de la Región de La Araucanía destaca por la presencia de humedales con vegetación boscosa de temu y pitra, de la asociación Blepharocalyo-Myrceugenietum exsuccae. Actualmente estas formaciones se encuentran afectadas por actividades antrópicas. En este estudio se determinó la flora vascular y se evaluó el nivel de degradación y estado de conservación de estos bosques en seis cuencas hidrográficas del borde costero (Imperial, Comúe, Chelle, Boroa, Boldo y Toltén) de la Región de La Araucanía. Para determinar el estado de conservación se emplearon variables florísticas y territoriales, como la valoración de la influencia de los ecosistemas adyacentes sobre el bosque, la relación superficie/ cuenca y el grado de intervención antrópica. Se utilizó información cartográfica sobre la distribución y superficie de los bosques pantanosos, estableciéndose de esta forma el mosaico vegetacional y la diversidad de los principales usos de los suelos. Los resultados muestran que los principales uso del área son agrícola, ganadero y silvícola. La flora vascular de los bosques la componen 94 especies, de las cuales el 14,9\% son introducidas, y el mayor porcentaje corresponde a nativas $(64,9 \%)$ y endémicas del país $(20,2 \%)$. Once especies se encuentran con problemas de conservación. Asimismo, todos los rodales presentan algún grado de degradación, siendo los ubicados en el curso inferior de la cuenca del río Imperial los que poseen el nivel más alto de degradación, y los menos intervenidos se encuentran en la cuenca del río Boldo. Se concluye que el estado de conservación promedio de los rodales en toda el área corresponde a "degradado". Como principal causa del deterioro están las actividades humanas realizadas tanto al interior como en la periferia de estos bosques.
\end{abstract}

Palabras clave: Conservación, humedal boscoso, Araucanía, Blepharocalyx, Myrceugenia.

\begin{abstract}
The coastal area of the Araucanía Region stands out for the presence of wooded wetlands of Temu and Pitra (BlepharocalyoMyrceugenietum exsuccae), from the association Blepharocalyo-Myrceugenietum exsuccae. These are nowadays affected by antropic activities. We evaluated the vascular flora, the degradation level and the condition of conservation of these forests, working in six basins of the coastal border (Imperial, Comúe, Chelle, Boroa, Boldo and Toltén) of the Araucanía Region. For determining the condition of conservation, different methods of evaluation were applied, using both floristic as well as territorial variables. Among these, the valuation of the influence of the adjacent ecosystems on the forest, the human intervention degree, and the relation between the surface of the forest and its basin. We used cartographic information about the distribution and surface of the swampy forest, setting this way the vegetation mosaic and the diversity of the main uses of the soils. The results show that the main uses of the area are agricultural, cattle and forestry activities. The vascular flora of the area consists of 94 species, $14.9 \%$ of them are introduced and the major percentage corresponds to natives from Chile and Argentina (64.9\%) and endemics of the country (20.2\%). Eleven species have conservation problems. At the same time, all the stands show some deterioration degree, being the stands with the highest level of deterioration those located in the basin of the Imperial river, unlike those located in the basin of the Boldo river, that have not been so damaged. We conclude that the average conservation condition of the stands of the whole studied area corresponds to "degraded", due to the effect of the different human activities carried out in these forests and on the periphery of them as well.
\end{abstract}

KeYwords: Conservation, swampy forest, Araucanía, Blepharocalyx, Myrceugenia. 


\section{INTRODUCCIÓN}

Los humedales son ecosistemas de un alto valor ecológico y los de mayor riqueza biológica del planeta(Kusler et al. 1994, Innis et al. 2000). Entre sus variadas funciones destaca su contribución en los ciclos de vida de plantas y animales, proveyendo de hábitat, alimento, sitios de nidificación y refugio para numerosas especies de fauna silvestre (CorreaAraneda et al. 2011). Moderan también los cambios climáticos, actúan como sumideros de $\mathrm{CO}_{2}$, amortiguan el efecto de las olas y almacenan las aguas de inundación, retienen el sedimento y reducen la contaminación. De igual forma, son importantes en la producción de alimentos y de forraje para animales domésticos y silvestres, y son fuente de materia prima para artesanía (Kusler et al. 1994, Hernández \& Sánchez 2009).

La presencia de humedales en la Región de La Araucanía, especialmente en su borde costero, se vio acrecentada con la formación de nuevas áreas inundadas por los efectos del terremoto y tsunami de mayo de 1960, constituyendo hoy día un gran potencial ambiental (Peña-Cortés et al. 2006). La permanencia de estos humedales crea la necesidad de conservar el recurso de agua dulce y su diversidad biológica asociada, donde la vida silvestre constituye un importante patrimonio natural, por lo que hoy son altamente valorados para las actividades turísticas y de recreación (CONAMA 2007). Según CONAF \& CONAMA (1999), los humedales de la región de La Araucanía abarcan el 5,1\% (23.138 ha) de la superficie regional; sólo en la provincia de Cautín se encuentran 17.625,2 ha con humedales palustres, ribereños y lacustres, de los cuales el 35,8\% se localiza en el borde costero de las comunas de Carahue, Saavedra, Teodoro Schmidt y Toltén.

A pesar de los múltiples beneficios directos y otros intangibles que aportan estos ecosistemas, la mayoría están expuestos a impactos ambientales. Su poca valoración como "lugares inservibles", es una barrera para la ejecución de proyectos de desarrollo y de conservación (Hauenstein et al. 2005).

Los bosques pantanosos de Chile se clasifican como humedales boscosos de agua dulce y su distribución es amplia y fragmentada, desde Coquimbo a Chiloé $\left(30^{\circ}\right.$ a

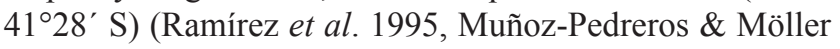
1997). La ubicación de preferencia es en depresiones con fallas tectónicas, depresiones interdunarias o en el fondo de valles o lechos fluviales, en sitios con escaso drenaje e inundaciones estacionales (Peña-Cortés et al. 2011). Estas características geomorfológicas facilitan el influjo constante de aguas subterráneas y superficialidad de la napa, configurando una oferta de hábitat para este tipo de vegetación azonal, con un carácter atípico para la zona. Sus taxa arbóreos dominantes son de géneros de las Myrtaceae (Luma, Blepharocalyx, Myrcengenia, Tepualia), en un sustrato en que soportan periodos de seis a 12 meses de inundación (Correa-Araneda et al. 2011, 2012).

Ramírez et al. (1995) describe estos bosques como formaciones de carácter azonal, con presencia dependiente de un exceso de humedad edáfica y no del macroclima. En relación con las asociaciones hasta hoy se han descrito las siguientes seis comunidades: a) de canelo, pitra y chequén (Lumo-Myrceugenietum exsuccae), b) de canelo, lingue y pitra (Perseo-Myrcengenietum exsuccae), c) de arrayán (Myrceugenielletum o Lumetum), d) de temo y pitra (Blepharocalyo-Myrceugenietum exsuccae), e) de tepú (Tepualietum stipulariae) y f) de chin-chin y canelo (Caldcluvio-Lumetum gayanae) (San Martín et al. 2008, Correa-Araneda et al. 2011).

En la Región de La Araucanía, el bosque pantanoso se presenta distribuido en fragmentos de formas muy irregulares insertos en una matriz agropecuaria (Peña-Cortés et al. 2011). Dentro de la diversidad de las asociaciones destaca el bosque pantanoso de temu Blepharocalyx cruckshanksii (Hook. \& Arn.) Nied. y pitra Myrceugenia exsucca (DC.) Berg, en especial los del sector Mahuidanchi-Lastarria, propuesto como sitio prioritario para la conservación de la diversidad biológica (CONAMA 2007). Sin embargo estos bosques son intervenidos a través del drenaje de sus suelos, de la tala y el roce a fuego, con el objeto de obtener suelos aptos para la agricultura y la reforestación con especies exóticas (Hauenstein et al.2005).

La eliminación de este tipo de humedal boscoso, como ya ha ocurrido en la Región del Maule (San Martín et al. 1990), significará una pérdida importante para la biodiversidad de la región de La Araucanía, sin haberse explorado previamente sus potencialidades como recurso natural, en actividades de desarrollo de las comunidades humanas aledañas, como por ejemplo el eco y etnoturismo. Desde el punto de vista ecológico, Ramírez et al. (1983, 1995), Glade (1987), Medina-Vogel (2002), González et al. (2003) y Correa-Araneda et al. (2011) señalan que este tipo de bosque representan un hábitat para la nidificación y refugio de especies con problemas de conservación como el huillín o nutria de río (Lontra provocax Thomas), torcaza (Patagioenas araucana Lesson) y cuervo del pantano (Plegadis chihi Veillot). Asimismo, desde la cosmovisión y etnia mapuche estos humedales son espacios sagrados y respetados, donde residen energías o fuerzas naturales y espirituales (newen y ñeh) y fuente de plantas medicinales, siendo denominados como "hualves o menoko" (Durán et al. 1997).

El objetivo de este trabajo es evaluarla flora y el estado de conservación de los rodales de bosque pantanoso de temo-pitra (Blepharocalyo-Myrceugenietum exsuccae), ubicados en el borde costero de la Región de La Araucanía. Estos antecedentes facilitarán la planificación de medidas para futuras investigaciones y programas de conservación, restauración o manejo. 


\section{MATERIALES Y MÉTODOS}

Área De ESTUdio

El estudio se realizó entre los años 2005 y 2010 en los periodos de primavera-verano en el borde costero de la Región de La Araucanía, Chile, entre las coordenadas $38^{\circ} 30^{\prime}-39^{\circ} 30^{\prime} \mathrm{S}$ y $72^{\circ} 45^{\prime}-73^{\circ} 30^{\prime} \mathrm{O}$. La superficie aproximada del área costera es de 166.589 ha, con las comunas de Carahue, Saavedra, Teodoro Schmidt y Toltén (Fig. 1), representando el 10,2\% de la superficie regional (Peña-Cortés et al. 2011). El clima de la zona es oceánico con influencia mediterránea, con una temperatura promedio anual de $12,3{ }^{\circ} \mathrm{C}$ y precipitación media anual de $1.182 \mathrm{~mm}$ (datos correspondientes a Puerto Saavedra) (Luebert \& Pliscoff 2006). Los suelos rojo-arcillosos más representativos corresponden a la asociación Nahuelbuta (Rhodic Paleudults, orden Ultisol) y serie Puerto Saavedra (Eutric Fulvudans, orden Andisol), existiendo otros con desarrollo incipiente y con limitante de uso a causa del drenaje imperfecto, que es donde se desarrolla preferentemente el bosque pantanoso (CIREN \& CORFO 1989).

\section{Metodología}

Sobre la base del estudio de Peña-Cortés et al. (2011), se delimitaron sectores de la zona costera con bosques pantanosos de temu y pitra, identificados con apoyo de fotografías aéreas, escala 1:20.000 años 2007-2009, y el uso de mapas topográficos del Instituto Geográfico Militar
(IGM, 1968-2000), escala 1:25.000. Con esta información se delimitaron cuencas y subcuencas de los ríos: Imperial, Comúe, Chelle, Boroa, Boldo y Toltén, basados en los límites de las líneas divisorias de aguas.

Para determinar la estructura y diversidad florísticovegetacional de los rodales en las seis cuencas se establecieron 11 estaciones de muestreo y 34 inventarios fitosociológicos de $100 \mathrm{~m}^{2}$ de superficie cada uno (BraunBlanquet 1979). La superficie representa réplicas superiores al área mínima (Steubing et al. 2002). Para cada especie se consideró su forma de vida de acuerdo a Ellenberg \& Mueller-Dombois (1966), su origen fitogeográfico según Matthei (1995), Zuloaga et al. (2008) y Marticorena et al. (2010) para trepadoras, epífitas y parásitas. El estado de conservación sigue a las listas oficiales del sistema de clasificación de especies del Ministerio del Medio Ambiente en sus nueve procesos (Dcto. Supremo 1971), así como otras sugeridas por Benoit (1989) y Rodríguez et al. (2009) para pteridófitos. La nomenclatura de las especies se basa en Zuloaga et al. (2008) y en las páginas web: (http://www.ipni.org/) y (http://www.darwin.edu. ar/). Con los resultados se elaboró un listado florístico general de los rodales de bosques pantanosos del borde costero (Anexo 1) y otro con la distribución espacial de las especies en cada sector (Anexo 2). Los ejemplares recolectados se depositaron en el Herbario de la Escuela de Ciencias Ambientales (UCT - Herbario no oficial), Facultad de Recursos Naturales, Universidad Católica

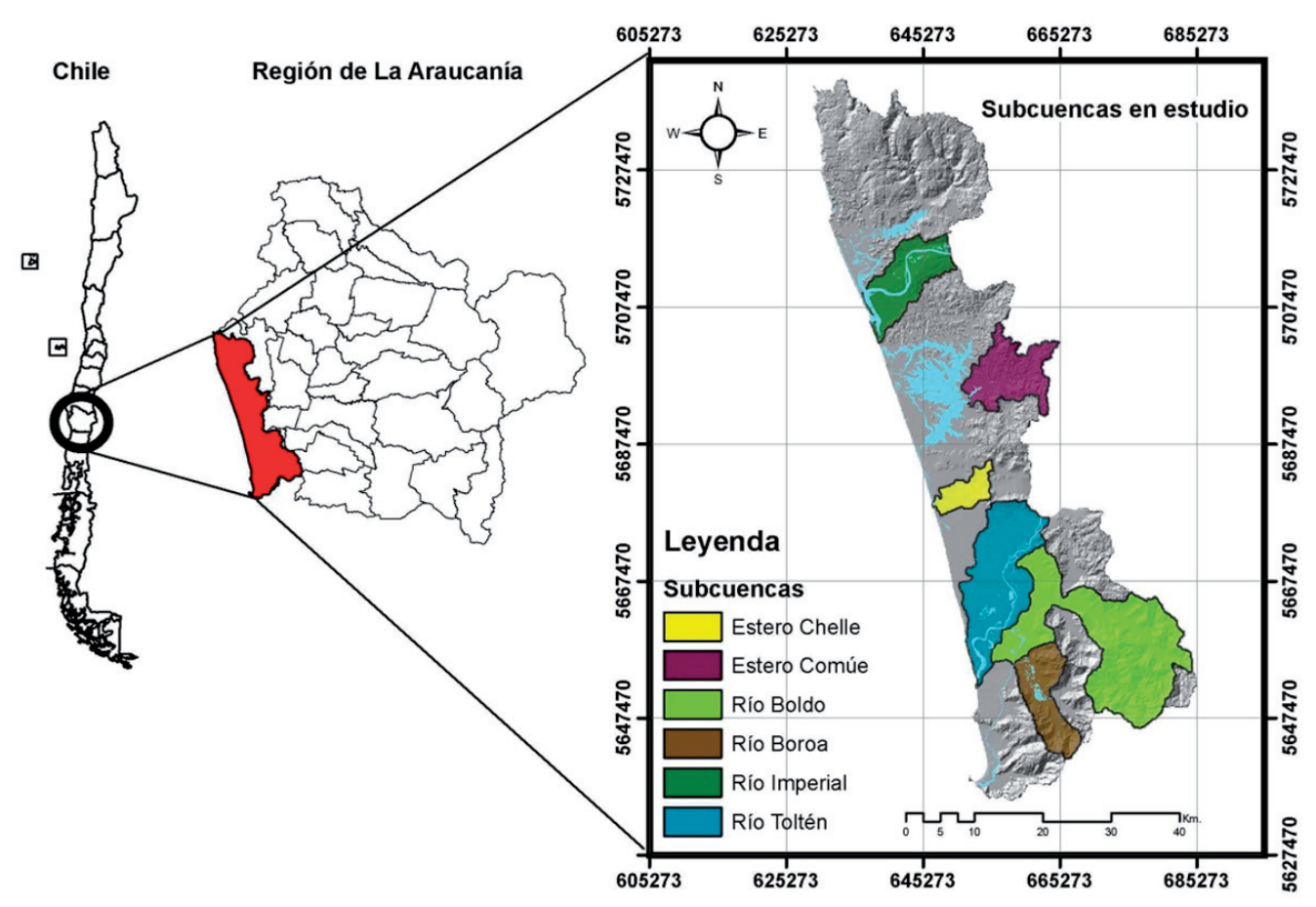

FIGURA 1. Ubicación geográfica del área de estudio y cuencas hidrográficas estudiadas.

FIGURE 1. Location of the study area and the hydrographic studied basins. 
de Temuco. Adicionalmente y para detectar similitudes florísticas se aplicó un análisis de Bray-Curtis, utilizando Biodiversity-Pro 2.0 (McAleece 1997).

Para la determinación del estado de conservación del bosque pantanoso se emplearon tanto variables florísticas como territoriales, estableciéndose niveles de deterioro y estado de conservación, mediante metodología EULA \& CONAMA (1999) modificada. La secuencia de las variables se ilustra en la Tabla I.

Así, el nivel de deterioro del bosque se expresa mediante la siguiente fórmula:

$\mathrm{ND}_{\mathrm{i}}=\Sigma\left(\left(\mathrm{EP}_{\mathrm{i}}^{*} \mathrm{WEP}\right)+\left(\mathrm{GI}_{\mathrm{i}}^{*} \mathrm{WGI}\right)+\left(\mathrm{SB}_{\mathrm{i}}^{*} \mathrm{WSB}\right)\right)$

Donde:

$\mathrm{ND}_{\mathrm{i}}=$ Nivel de deterioro del bosque en la cuenca. $\mathrm{EP}_{\mathrm{i}}=$ Ecosistemas próximos al humedal en la cuenca. $\mathrm{GI}_{\mathrm{i}}=$ Grado de intervención antrópica del bosque. $\mathrm{SB}_{\mathrm{i}}=$ Superficie del bosque respecto a su cuenca. $\mathrm{WEP}=$ peso de la variable EP, la que corresponde a 0,4 . WGI $=$ peso de la variable GI, la que corresponde a $0,2 . \mathrm{WSB}=$ peso de la variable $\mathrm{SB}$, la que corresponde a 0,4 .
Los resultados obtenidos se clasificaron de acuerdo a niveles de deterioro, los que a su vez se traducen en categorías del estado de conservación de los rodales, como se indica en la Tabla II.

\section{RESULTADOS}

\section{CARACTERIZACIÓN CUENCAS HIDROGRÁFICAS}

Las cuencas estudiadas (Tabla III) comprenden aproximadamente 77.591 ha, siendo la mayor superficie para el río Toltén con 25.090 ha y la menor el río Imperial con 6.273 ha. Las seis cuencas se caracterizan por su baja altitud, no superando los $40 \mathrm{~m}$ de elevación.

En ellas, el área aproximada ocupada por el bosque pantanoso es de 4.574 ha, correspondiendo al 5,9\% de la superficie total de éstas. La cuenca con los mayores porcentajes de bosque en relación a su superficie es la del río Boldo con un 9\%. La morfología de las cuencas está dominada por cordones montañosos, plataformas y llanuras fluviomarinas; el uso de suelo corresponde principalmente a praderas de uso agrícola, ganadero y silvícola.

TABLA I. Variables consideradas y escala de valoración correspondiente.

TABLE I. Considered variables and corresponding rating scale.

\begin{tabular}{ll}
\hline VARIABLES & ESCALA DE VALORACIÓN \\
\hline Ecosistemas próximos al rodal & $0=$ humedales, boscosos \\
& $2=$ ambientes litorales \\
& $10=$ agrícolas, ganaderos, forestales. \\
Grado de perturbación antrópica & $2=$ poco o nulo \\
& $5=$ medianamente intervenido \\
& $10=$ altamente intervenido. \\
Superficie del bosque respecto de su cuenca & $2=\geq 10 \%$ \\
& $5=\geq 5 \% \mathrm{y}<10 \%$ \\
& $10=<5 \%$ \\
\hline
\end{tabular}

TABLA II. Escala de valoración de los niveles de deterioro y estado de conservación.

TABLE II. Scale of valuation of the levels of deterioration and condition of conservation.

\begin{tabular}{cll}
\hline Rangos Valores & Nivel Deterioro & Estado Conservación \\
\hline$\geq 8,6$ & Muy alto & Muy degradado \\
$7,2-8,5$ & Alto & Degradado \\
$5,7-7,1$ & Moderadamente alto & Medianamente degradado \\
$4,2-5,6$ & Moderadamente bajo & Escasamente degradado \\
$2,8-4,1$ & Bajo & Casi sin degradación \\
$\leq 2,7$ & Muy bajo & Sin degradación \\
\hline
\end{tabular}


Florística y degradación del bosque pantanoso: HaUENSTEIN, E. ET AL.

TABLA III. Resumen de indicadores descriptivos de las cuencas hidrográficas estudiadas en el borde costero de la Región de La Araucanía con estaciones de muestreo, posición geográfica, altitud y relevamientos.

TABLE III. Summary of descriptive indicators of the hydrographic basins studied in the coastal edge of the Araucania Region with sampling stations, geographical position, altitude and sensuses.

\begin{tabular}{|c|c|c|c|c|c|c|}
\hline Cuenca & AREA (ha) & Uso de Suelo & EstACIONES & COORdenadas & $\begin{array}{l}\text { ALtiTUD } \\
(\mathrm{msnm}) \\
\end{array}$ & $\mathrm{N}^{\circ}$ \\
\hline Imperial & 6.273 & $\begin{array}{l}\text { Rotación cultivo- } \\
\text { pradera }\end{array}$ & $\begin{array}{l}\text { Cruce puente } \\
\text { Nehuentue }\end{array}$ & $38^{\circ} 42^{\prime} 45,2^{\prime \prime} \mathrm{S} ; 73^{\circ} 20^{\prime} 8,6^{\prime \prime} \mathrm{O}$ & 10 & 3 \\
\hline \multirow{2}{*}{ Comúe } & \multirow{2}{*}{8.783} & \multirow{2}{*}{$\begin{array}{l}\text { Cultivo agrícola } \\
\text { tradicional }\end{array}$} & Estero Comúe 1 & $38^{\circ} 52^{\prime} 14,3^{\prime \prime} \mathrm{S} ; 73^{\circ} 13^{\prime} 15,3^{\prime \prime} \mathrm{O}$ & 24 & 3 \\
\hline & & & Estero Comúe 2 & $38^{\circ} 52^{\prime} 25,7^{\prime \prime} \mathrm{S} ; 73^{\circ} 13^{\prime} 32,5^{\prime \prime} \mathrm{O}$ & 12 & 3 \\
\hline \multirow{2}{*}{ Chelle } & \multirow{2}{*}{9.693} & \multirow{2}{*}{$\begin{array}{l}\text { Rotación cultivo- } \\
\text { pradera }\end{array}$} & Estero Chelle & $39^{\circ} 00^{\prime} 26,1^{\prime \prime} \mathrm{S} ; 3^{\circ} 15^{\prime} 23,1^{\prime \prime} \mathrm{O}$ & 0 & 4 \\
\hline & & & Estero Tricauco & $39^{\circ} 03^{\prime} 50,8^{\prime \prime} \mathrm{S} ; 73^{\circ} 09^{\prime} 38,3^{\prime \prime} \mathrm{O}$ & 5 & 3 \\
\hline \multirow{2}{*}{ Boroa } & \multirow{2}{*}{13.342} & \multirow{2}{*}{$\begin{array}{l}\text { Plantaciones y } \\
\text { praderas perennes }\end{array}$} & Boroa central & $39^{\circ} 17^{\prime} 24,9^{\prime \prime} \mathrm{S} ; 73^{\circ} 04^{\prime} 48,5^{\prime \prime} \mathrm{O}$ & 25 & 2 \\
\hline & & & Boroa sur & $39^{\circ} 20^{\prime} 15,0^{\prime \prime} \mathrm{S} ; 73^{\circ} 04^{\prime} 25,8^{\prime \prime} \mathrm{O}$ & 20 & 7 \\
\hline \multirow{2}{*}{ Boldo } & \multirow{2}{*}{14.410} & \multirow{2}{*}{$\begin{array}{l}\text { Plantaciones y } \\
\text { praderas perennes }\end{array}$} & San Roque alto & $39^{\circ} 09^{\prime} 56,3^{\prime \prime} \mathrm{S} ; 73^{\circ} 00^{\prime} 32,8^{\prime \prime} \mathrm{O}$ & 40 & 2 \\
\hline & & & San Roque bajo & $39^{\circ} 09^{\prime} 14,3^{\prime \prime} \mathrm{S} ; 73^{\circ} 03^{\prime} 0,9^{\prime \prime} \mathrm{O}$ & 10 & 2 \\
\hline \multirow{2}{*}{ Toltén } & \multirow{2}{*}{25.090} & \multirow{2}{*}{$\begin{array}{l}\text { Rotación cultivo- } \\
\text { pradera }\end{array}$} & Punta de rieles & $39^{\circ} 12^{\prime} 6,9^{\prime \prime} \mathrm{S} ; 73^{\circ} 13^{\prime} 46,7^{\prime \prime} \mathrm{O}$ & 1 & 3 \\
\hline & & & Toltén viejo & $39^{\circ} 11^{\prime} 26,2^{\prime \prime} \mathrm{S} ; 73^{\circ} 11^{\prime} 18,1^{\prime \prime} \mathrm{O}$ & -3 & 2 \\
\hline
\end{tabular}

\section{RiQueZa TAXONÓMICA}

Se registró un total de 94 especies vasculares (Anexo 1, Tabla IV). Las Magnoliopsida (dicotiledóneas) con 60 especies (63,8\%), Liliopsida (monocotiledóneas) 15 $(16,0 \%)$ y 19 pteridófitos (20,2\%). No se registraron gimnospermas. La distribución de la riqueza en los rodales se muestra en el Anexo 2. Los rodales de Imperial, Comúe y Boroa presentaron el menor número de especies (23, 24 y 30 respectivamente), contrariamente Chelle, Toltén y Boldo el mayor número (51, 55 y 61 spp.); asimismo, las especies con mayor fidelidad en los rodales por forma de crecimiento fueron tres arbóreas (Blepharocalyx cruckshanksii (Hook. \& Arn.) Nied., Drimys winteri J.R. Forster \& G. Forster, Myrceugenia exsucca (DC.) O.Berg), dos hierbas robustas (Blechnum chilense (Kaulf.) Mett., Cyperus eragrostis Lam.), una arbustiva (Rubus constrictus P.J. Müll. \& Léfebre) y tres trepadoras (Boquila trifoliolata (DC.) Decne., Cissus striata Ruiz \& Pav., Muehlenbeckia hastulata (Sm.) I.M.Johnst.).

El índice de similitud florística (Fig. 2) muestra tres conglomerados: el primero reúne los stands de las cuencas de los ríos Boldo-Toltén, con la mayor similitud de 65,0\%. Le siguen los rodales de Chelle-Comúe y Boroa-Chelle con
$59,3 \%$ y $58,8 \%$, respectivamente. Los rodales de la cuenca del río Imperial se segregan con una similitud menor.

ORIGEN FITOGEOGRÁFICO Y FORMAS DE VIDA

El origen fitogeográfico de las especies en los rodales (Tabla V) registra 56 especies nativas $(59,6 \%), 26$ endémicas $(27,6 \%)$ y 12 introducidas $(12,8 \%)$. Los resultados por cuencas, indican que en los rodales de los ríos Boroa, Boldo y Chelle el 90\% corresponden a especies nativas-endémicas y valores inferiores a 10\% de alóctonas. En cambio en los rodales de Imperial, Comúe y Toltén los porcentajes de especies nativas-endémicas oscilan entre 80 y $86 \%$, siendo los de Imperial los que presentan el porcentaje mayor de especies introducidas (17,9\%).

Las formas de vida están representadas en las cinco formas propuestas por Raunkiaer (1937) (Figura 3), y en orden decreciente los resultados fueron: hemicriptófitos con 23 especies $(24,5 \%)$ como grupo predominante, fanerófitos y epífitos con 17 y 15 especies respectivamente (18,1 y 16,0\%), criptófitos y nanofanerófitos con 11 $(11,7 \%)$ cada uno, las lianas con nueve especies $(9,6 \%)$, parásitos con cuatro $(4,2 \%)$, caméfitos y terófitos con dos $(2,1 \%)$ cada uno. 
Con problemas de conservación se registraron 12 especies $(12,8 \%)$ (Tabla VI), distribuidas en ocho pteridófitos, una dicotiledónea (Blepharocalyx cruckshanksii) y tres monocotiledóneas (Fascicularia bicolor (Ruiz \& Pav.) Mez, Greigia sphacelata Regel y Lapageria rosea Ruiz \& Pav.). De ellas, ocho están en la condición de Vulnerables, una con Datos insuficientes, una Casi amenazada, una en Preocupación menor y una en Peligro.

ESTADO DE CONSERVACIÓN DE LOS RODALES

La valoración final de los niveles de deterioro y estado de conservación de los rodales estudiados (Tabla VII), muestra que los del río Boldo presentan mejores condiciones, con un nivel de deterioro "moderadamente bajo" y estado de conservación "escasamente degradado". Por el contrario en los de la cuenca del río Imperial el deterioro es "muy alto" y el estado de conservación "muy degradado". Los rodales de las otras cuatro cuencas se encuentran en una situación intermedia, considerándose su estado de conservación entre degradado y medianamente degradado. En promedio para todos los rodales por perturbación ensu estructura y modificación en la composición, el nivel de deterioro es "alto" y el estado de conservación es "degradado".

TABLa IV. Distribución general de la flora vascular según categoría taxonómica en los rodales de bosque pantanoso.

TABLE IV. General distribution of the vascular flora according to taxonomic category in the stands of swampy forest.

\begin{tabular}{lcccr}
\hline Clases & Familias & GÉNEROS & EspeCies & $\%$ spp. \\
\hline Sphenopsida & 1 & 1 & 1 & 1,06 \\
Filicopsida & 6 & 7 & 18 & 19,15 \\
Magnoliopsida & 36 & 48 & 60 & 63,83 \\
Liliopsida & 7 & 13 & 15 & 15,96 \\
\hline Total & 50 & 69 & 94 & 100,0 \\
\hline
\end{tabular}

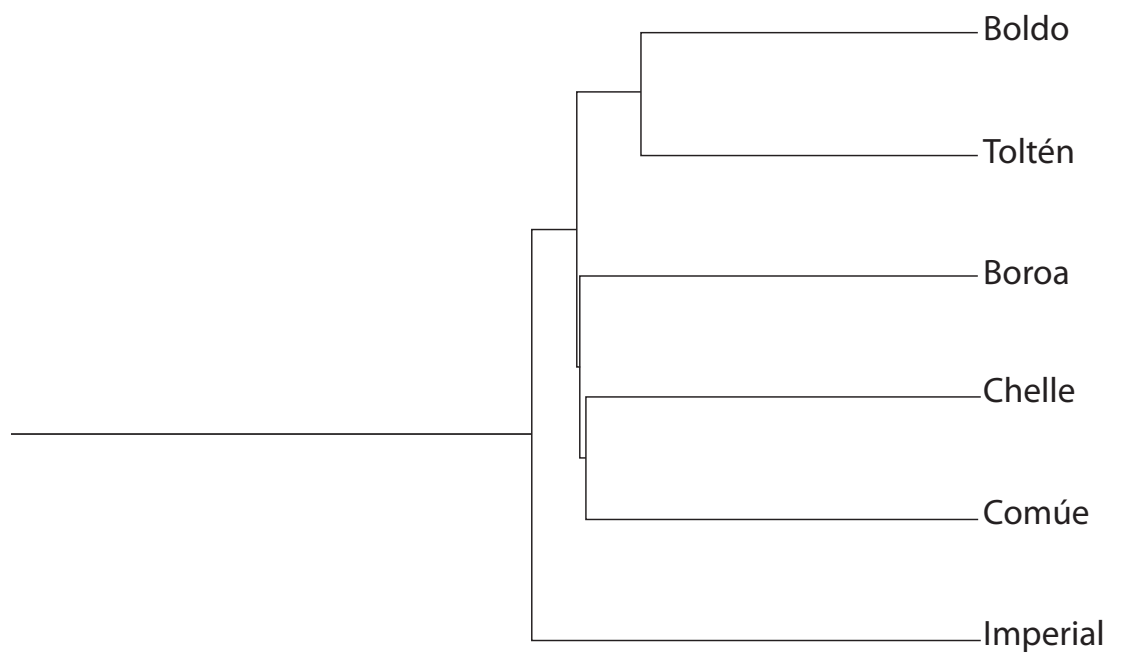

$0 \%$ Similarity

50 100

FIGURA 2. Dendrograma de similitud florística entre las cuencas estudiadas.

FIGURE 2. Dendrogram of floristic similarity between the studied basins. 
Florística y degradación del bosque pantanoso: HaUenstern, E. ET AL.

TABLA V. Origen fitogeográfico de las especies, ordenado según clases taxonómicas.

TABLE V. Phytogeographycal origin of the species, according to the taxonomic classes.

\begin{tabular}{|c|c|c|c|c|c|c|}
\hline Clase & Nativas & $\%$ & ENDÉMICAS & $\%$ & Alóctonas & $\%$ \\
\hline Sphenopsida & 1 & 1,06 & 0 & 0 & 0 & 0 \\
\hline Filicopsida & 14 & 14,89 & 4 & 4,25 & 0 & 0 \\
\hline Magnoliopsida & 34 & 36,17 & 15 & 15,95 & 11 & 11,70 \\
\hline Liliopsida & 7 & 7,45 & 7 & 7,45 & 1 & 1,06 \\
\hline Total & 56 & 59,57 & 26 & 27,65 & 12 & 12,76 \\
\hline
\end{tabular}

TABLA VI. Listado de especies con problemas de conservación y respectivos autores.

TABLE VI. List of species with conservation problems and respective authors.

\begin{tabular}{lll}
\hline EsPeCIES & $\begin{array}{l}\text { ESTADO DE } \\
\text { CONSERVACIÓN }\end{array}$ & Autor \\
\hline PTERIDOPHYTA & & \\
Asplenium trilobum & Vulnerable (VU) & \\
Grammitis magellanica & Vulnerable (VU) & Rodríguez et al. (2009) \\
Hymenoglossum cruentum & Vulnerable (VU) & Rodríguez et al. (2009) \\
Hymenophyllum caudiculatum & Vulnerable (VU) & Rodríguez et al. (2009) \\
Hymenophyllum dicranotrichum & Vulnerable (VU) & Rodríguez et al. (2009) \\
Hymenophyllum plicatum & Preocupación menor (I.C.) & Rodríguez et al. (2009) \\
Hymenophyllum tortuosum & Vulnerable (VU) & MMA (2013, 9 proceso) \\
Hypolepis poeppigii & Datos insuficientes (DD) & Rodríguez et al. (2009) \\
MAGnOLIOPHYTA & & Rodríguez et al. (2009) \\
Blepharocalyx cruckshanksii & Casi amenazada (NT) & \\
Fascicularia bicolor & Vulnerable (VU) & Rodríguez et al. (2005) \\
Greigia sphacelata & Vulnerable (VU) & Benoit (1989) \\
Lapageria rosea & En peligro (EN) & Benoit (1989) \\
\hline
\end{tabular}

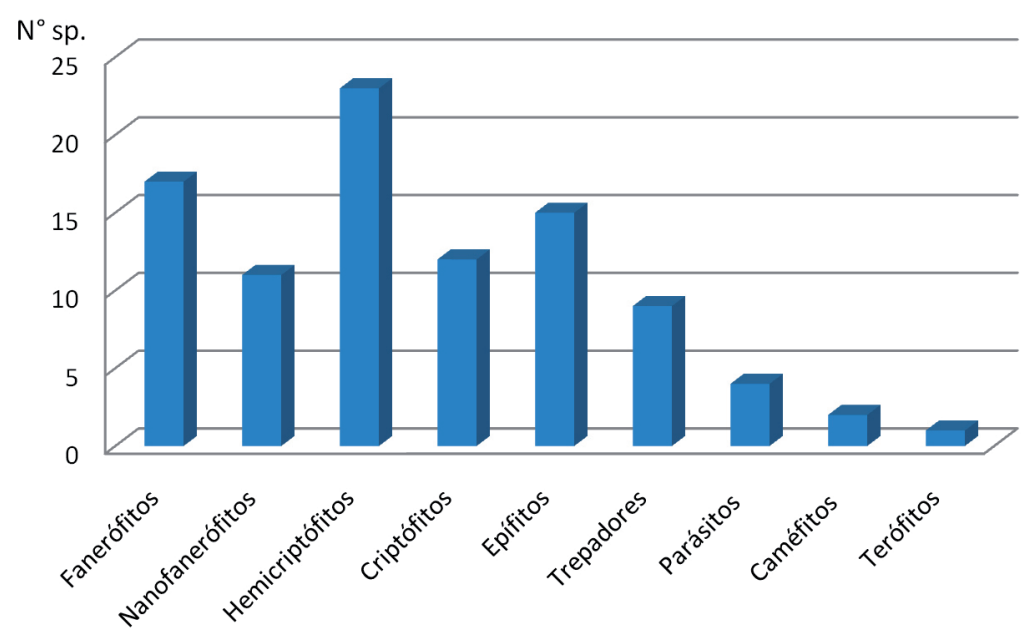

Figura 3. Formas de vida de la flora de los bosques pantanosos costeros de la Región de La Araucanía.

FIGURE 3. Life forms of the flora of swamp forests of the coast of the Araucania Region. 
TABLA VII. Nivel de deterioro y estado de conservación de los rodales de bosque pantanoso por cuenca hidrográfica, en la zona costera de la Región de La Araucanía, según escala de EULA \& CONAMA (1999) modificada.

TABLE VII. Level of deterioration and condition of conservation of the swampy forest stands for hydrographic basin, in the coastal zone of the Araucania Region, according to scale of EULA \& CONAMA (1999) modified.

\begin{tabular}{lcc}
\hline Cuenca & Nivel de Deterioro & Estado de Conservación \\
\hline Imperial & $(9,0)$ Muy Alto & Muy degradado \\
Comúe & $(8,4)$ Alto & Degradado \\
Boroa & $(8,4)$ Alto & Degradado \\
Chelle & $(6,4)$ Moderadamente alto & Medianamente degradado \\
Toltén & $(6,4)$ Moderadamente alto & Medianamente degradado \\
Boldo & $(5,2)$ Moderadamente bajo & Escasamente degradado \\
\hline Promedio & $(7,3)$ Alto & Degradado \\
\hline
\end{tabular}

\section{DISCUSIÓN}

Los usos de suelo en el área de estudio corresponden a cultivos y praderas de rotación, praderas perennes y plantaciones (Peña-Cortés et al. 2011). Estos ecosistemas artificiales componen la matriz que rodea a los rodales de bosque pantanoso, reflejando una relación directa con las principales causas de su fragmentación, como son el drenaje de los suelos para habilitar terrenos para cultivos agrícolas, especialmente de papas (Solanum tuberosum L.), praderas de pastoreo y extracción de leña (Peña-Cortés et al. 2006).

La actividad agrícola tiene sus orígenes en la región desde la colonización de la Araucanía y ha persistido hasta hoy con cambios en el régimen de tenencia de la propiedad, naturaleza e intensidad de cultivos, pastoreo y plantaciones (Gallegos 2009). Esta temporalidad en el uso y configuración de la matriz explica la fragmentación, reducción areal y vulnerabilidad de la vegetación higrófilapantanosa (Ramírez et al. 1995). Frente a esta presión ambiental se confirma que de la diversidad de especies arbóreas Blepharocalyx cruckshanksii es la especie mejor adaptada para sobrevivir en condiciones desfavorables, que se explica por la formación de raíces adventicias aéreas en el tronco durante los periodos de inundación (Weinberger et al. 1973).

Correa-Araneda et al. (2012) señalan que el factor hidroperiodo tiene un efecto importante en el desarrollo vegetativo de las especies de estos bosques y en la estructura de la comunidad. Los resultados concuerdan con lo señalado por el autor mencionado encontrándose, según el tipo de ambiente, diferentes formas de crecimiento: a) en inundación permanente (10-12 meses), los árboles crecen agrupados en "matas" con troncos retorcidos y de baja altura $(<12 \mathrm{~m}) ; \mathrm{b})$ en inundaciones periódicas (hasta 6 meses), los fustes crecen separados, rectos y de mayor altura (hasta 20 m). La mayoría de los rodales estudiados corresponden al primer tipo.
La importancia de la flora de los rodales radica fundamentalmente en la abundante presencia de epífitas (15 spp.), principalmente de la familia Hymenophyllaceae (9 spp.), lo que indica altos niveles de humedad al interior del bosque (Alberdi et al. 1978), y de trepadoras (9 spp.). Estos resultados concuerdan con Ramírez et al. (1976), quien registró nueve especies de Hymenophyllaceae para un bosque de olivillo (Aextoxicon punctatum Ruiz \& Pav.) cerca de Valdivia, discrepando con lo reportado por San Martín et al. (2008) para un bosque primario de olivillo, registrando sólo ocho especies de epífitas de las cuales seis eran Hymenophyllaceae. De igual forma Godoy et al. (1981), para comunidades boscosas de temo-pitra de la $\mathrm{X}$ Región, mencionan 13 especies de Pteridófitos, y en el presente estudio se registraron 19. Si bien los resultados tienen origen en comunidades y tiempos diferentes, la no coincidencia con nuestra información se explicaría por la modalidad de muestreo y focalización en el área costera así como la revisión exhaustiva de los rodales.

Desde el punto de vista de la conservación se destaca la presencia de 12 especies con problemas de conservación, con dominio nuevamente de las Hymenophyllaceae (5 spp.). Las especies de esta familia son exigentes en un microclima húmedo $\mathrm{y}$ de integridad del bosque como se describe para otros siempreverdes como el bosque de olivillo (Alberdi et al. 1978). Estos elementos refuerzan la tesis de focalizar los esfuerzos para su protección in situ (Benoit 1989, Ramírez et al. 1995, Rodríguez et al. 2009).

El origen fitogeográfico registra un $87,2 \%$ de especies nativas y endémicas contra un $12,8 \%$ de alóctonas. Como consecuencia de la apertura de los bosques, la relación de elementos autóctonos respecto a alóctonos se ha usado como un índice de degradación o invasión (Hauenstein et al. 1988, González 2000); considerando sólo esta relación para determinar la acción antrópica según la escala propuesta por González (2000), se encontró que en los bosques costeros los valores alcanzan entre 12 y $20 \%$ de especies introducidas, 
correspondiendo a un sistema "Poco Intervenido". Estos valores son inferiores respecto a lo señalado por Ramírez et al. (1995) para Chile sur-central, con un $61 \%$ de especies nativas y $39 \%$ de alóctonas. La magnitud de los valores para los bosques costeros es un indicador de que, a pesar de su tala indiscriminada, mantienen una composición florística conservada (Hauenstein et al. 2005, Hernández \& Sánchez 2009).

Los rodales de las cuencas de los ríos Boroa, Boldo y Chelle presentan sobre un $90 \%$ de especies nativasendémicas y valores de especies introducidas inferiores a $10 \%$, en cambio los del río Imperial presentan los mayores porcentajes de especies introducidas $(17,9 \%)$, lo cual reafirma su mayor grado de alteración.

Estos resultados, en conjunto con la similitud florística y los del espectro biológico (formas de vida) para los rodales de los ríos Boroa, Toltén y Chelle, que registraron los mayores porcentajes de hemicriptófitos, se explicaría por la mayor apertura del dosel arbóreo y el resultado está de acuerdo con lo sugerido por Hauenstein et al. (1988) y Grigera et al. (1996), al considerarlos como especies indicadoras de intervención antrópica. Asimismo, Ramírez et al. (2012) indica que si al origen geográfico de la flora se agregan las formas de vida, se obtiene una mayor aproximación, especialmente para identificar el factor causante del cambio antrópico y el nivel de degradación alcanzado.

En el dendrograma de similitud florística (Fig. 2) se aprecia que hay una afinidad espacial entre los sectores, ya que Toltén, Boldo y Boroa están geográficamente reunidos hacia el sur del territorio, en cambio Chelle, Comúe e Imperial se ubican hacia el norte, coincidiendo con lo señalado por Peña-Cortés et al. $(2006,2011)$ respecto de que en el sector norte existe una mayor división predial y número de caminos interiores, lo que explica una mayor antropización y por ende la presencia de una mayor cantidad de especies alóctonas, que explicarían dichas diferencias.

Por otra parte, es importante el alto número de especies epífitas y trepadoras de estos rodales, y de cuatro hemiparásitos vasculares, donde destaca por su abundancia Lepidoceras chilense (Molina) Kuijt.

Si bien los niveles de degradación indican que los bosques pantanosos del río Imperial son los más degradados (Tabla VII), los de Chelle, Comúe, Boroa y Toltén están en un grado intermedio y los del río Boldo como los más prístinos. En el área todos los rodales están expuestos a la acción humana, la que en los últimos años se ha intensificado. Una variable que atenta contra la integridad de los bosques es el desecamiento con drenaje que acelera la habilitación de los suelos para uso agrícola. También se agrega la tala constante y la sustitución por especies forestales de Eucalyptus y Populus (Hauenstein et al. 2005). Estos resultados son congruentes con los de Peña-Cortés et al. (2011) que indican que el 40\% del bosque se localiza hasta $300 \mathrm{~m}$ de distancia de caminos y sólo el $8 \%$ se ubica a más de $1.000 \mathrm{~m}$, y su tamaño predial está asociado preferentemente a pequeños productores que requieren hacer productivo el recurso suelo.

Además de los elementos florísticos y estructurales del bosque es importante incluir los ecosistemas asociados, ya que se consideran fuente de elementos invasores como introducción de malezas o especies foráneas, muchas de ellas con potencialidad de desplazar a las propias del bosque (Fuentes-Ramírez et al. 2011). La supervivencia e integridad de estos bosques se relaciona con la capacidad y estrategia reproductiva de las especies arbóreas, demostrada por Hechenleitner et al. (2005) y Latsague et al. (2010) que han determinado la capacidad germinativa de las semillas y su propagación vegetativa.

\section{AGRADECIMIENTOS}

A los proyectos FONDECYT 1080317 y FONDECYT 1110798 "Determinación de indicadores geográficoambientales y de riesgo natural en el paisaje de La Araucanía y Los Ríos: Herramientas de soporte decisional para la planificación y gestión territorial en sistemas costeros", y DGIP-UCT 2008-3-01, de la Dirección de Investigación y Postgrado de la Universidad Católica de Temuco.

\section{BIBLIOGRAFÍA}

Alberdi, M., C. Ramírez \& L. Steubing. 1978. La familia Hymenophyllaceae (Pteridophyta) en el fundo San Martín, Valdivia, Chile. II. Resistencia al desecamiento y sobrevivencia en comunidades antropogénicas. Medio Ambiente 3(2): 3-13.

Benoit, I.L. (ed.) 1989. Libro rojo de la flora terrestre de Chile. Corporación Nacional Forestal(CONAF), Santiago, Chile. $157 \mathrm{pp}$.

Braun-Blanquet, J. 1979. Fitosociología. Base para el estudio de las comunidades vegetales. Editorial Blume. Madrid, España. 820 pp.

Centro de Información de Recursos Naturales (CIREN) \& Corporación de Fomento de la Producción (CORFO). 1989. Estudio agroecológico de la provincia de Cautín. Descripciones de suelos, materiales y símbolos IX Región. Publicación $N^{\circ} 77.360$ pp.

Centro Universitario Internacional Europa Latinoamérica (EUlA) \& Comisión Nacional del Medio Ambiente (CONAMA). 1999. Orientación para la evaluación de impactos ambientales: Industria de la madera. Ed. Centro Universitario Internacional Europa Latinoamérica Comisión Nacional del Medio Ambiente, Chile. 238 pp.

Comisión Nacional del Medio Ambiente (CONAMA). 2007. Plan de acción de biodiversidad 2007-2015. Comité Operativo de Biodiversidad, Región de La Araucanía, Chile. 28 pp.

Corporación Nacional Forestal (CONAF) \& Comisión Nacional del Medio Ambiente (CONAMA). 1999.Proyecto catastro y evaluación de recursos vegetacionales nativos de Chile. Informe Regional Novena Región. Santiago, Chile. 90 pp. 
Correa-Araneda, F., J. Urrutia \& R. Figueroa. 2011. Estado del conocimiento y principales amenazas de los humedales boscosos de agua dulce de Chile. Revista Chilena de Historia Natural 84: 325-340.

Correa-Araneda, F., J. Urrutia, Y. Soto-Mora, R. Figueroa \& E. Hauenstein. 2012. Effects of the hydroperiod on the vegetative and community structure of freshwater forested wetlands, Chile. Journal of Freshwater Ecology 27(3): 459-470.

Durán, T., J. Quidel \& E. Hauenstein. 1997. Conocimientos y vivencias de dos familias Wenteche sobre medicina mapuche. Centro de Estudios Socio-Culturales, Universidad Católica de Temuco-Chile y Ediciones LOM. 99 pp.

EllenberG, H. \& D. Mueller-Dombois. 1966. A key to Raunkiaer plant life forms with revised subdivisions. Berein Geobotanik Institut ETH Stiffung Rübel, Zurich 37: 56-73.

Fuentes-Ramírez, A., A. Pauchard, L.A. Cavieres \& R.A. García. 2011. Survival and growth of Acacia dealbata vs. native trees across an invasion front in south-central Chile. Forest Ecology and Management 261: 1003-1009.

Gallegos, S. 2009. Antecedentes históricos y de evolución ambiental del borde costero de la Región de La Araucanía. Tesis Licenciatura en Recursos Naturales. Escuela de Ciencias Ambientales, Universidad Católica de Temuco, Chile. 163 pp.

Glade, A. 1987. Libro rojo de los vertebrados terrestres de Chile. Corporación Nacional Forestal (CONAF), Santiago, Chile. $65 \mathrm{pp}$.

Godoy, R., C. Ramírez, H. Figueroa \& E. Hauenstein. 1981. Estudios ecosociológicos en pteridófitos de comunidades boscosas valdivianas, Chile. Bosque 4(1): 12-24.

GonzÁlez, A. 2000. Evaluación del recurso vegetacional en la cuenca del río Budi, situación actual y propuestas de manejo. Tesis Licenciatura en Recursos Naturales. Escuela de Ciencias Ambientales, Facultad de Recursos Naturales, Universidad Católica de Temuco, Temuco, Chile. 87 pp.

González, M., E. Hauenstein, F. Peña-Cortés, M. García \& O. URrutia. 2003. Comentarios sobre bosques pantanosos, humedales importantes del Centro-Sur de Chile. Gestión Ambiental 9: 3-13.

Grigera, D., C. Brion, J.O. Chiapella \& M.S. Pillado. 1996. Las formas de vida de las plantas como indicadores de factores ambientales. Medio Ambiente 13(1):11-29.

Hauenstein, E., C. Ramírez, M. Latsague \& D. Contreras. 1988. Origen fitogeográfico y espectro biológico como medida del grado de intervención antrópica en comunidades vegetales. Medio Ambiente 9(1): 140-142.

Hauenstein, E., M. González, F. Peña-Cortés \& A. MuñozPedreros. 2005. Diversidad vegetal en humedales costeros de la Región de La Araucanía. En: C. Smith-Ramírez, J.J. Armesto \& C. Valdovinos (eds.), Historia, biodiversidad y ecología de los bosques costeros de Chile, pp. 197-205. Editorial Universitaria, Santiago, Chile.

Hechenleitner, P., M. Gardner, P. Thomas, C. Echeverría, B. Escobar, P. Brownless \& E. Martínez. 2005. Plantas amenazadas del Centro Sur de Chile. Distribución, conservación y propagación. Valdivia, Chile. Universidad Austral de Chile - Real Jardín Botánico de Edimburgo. 188 pp.
Hernández, M. \& P. SÁnchez (eds.). 2009. Humedales, espacios para la conservación de la biodiversidad en la región de La Araucanía, Chile. 81 pp.

Hoffmann, A.E. 1991. Flora Silvestre de Chile. Zona Araucana. $2^{\text {a }}$ ed. Ediciones Claudio Gay. Santiago, Chile. 257 pp.

InNis, S., R. NAIMAN \& S. ELLIOTt. 2000. Indicators and assessment methods for measuring the ecological integrity of semiaquatic terrestrial environments. Hydrobiología 422/423: 111-131.

Kusler, J., W. Mitsch \& J. LARSON. 1994. Humedales. Investigación y Ciencia 210: 6-13.

Latsague, M., P. Sáez, E. Hauenstein \& F. Peña-Cortés. 2010. Propagación vegetativa de Myrcengenia exsucca y Blepharocalyx cruckshanksii, especies dominantes del bosque pantanoso de la Depresión Intermedia de la Región de La Araucanía, Chile. Bosque 31(3): 247-251.

Luebert, F. \& P. Pliscoff. 2006. Sinopsis bioclimática y vegetacional de Chile. Editorial Universitaria. Santiago, Chile. 316 pp.

Marticorena, A., D. Alarcón, L. Abello \& C. Atala. 2010. Plantas trepadoras, epífitas y parásitas nativas de Chile. Guía de Campo. Ed. Corporación Chilena de la Madera (CORMA), Concepción, Chile. 291 pp.

Matthei, O. 1995.Manual de las malezas que crecen en Chile. Alfabeta Impresores, Santiago, Chile. 545 pp.

McAleece, N. 1997. Biodiversity Professional Beta 1. Version 2.0. The Natural History Museum and the Scottish Association for Marine Science.

Medina-Vogel, G. 2002. Visión ecorregional para la conservación del Huillín (Lontra provocax) en Chile. Instituto de Ecología y Evolución, Universidad Austral de Chile, Valdivia. $28 \mathrm{pp}$.

Muñoz-Pedreros, A. \& P. Möller (eds.) 1997. Conservación de humedales. Taller bases para la conservación de humedales de Chile. CEA Ediciones, Valdivia. 95 pp.

Peña-Cortés, F., P. Gutiérrez, G. Rebolledo, M. Escalona, E. Hauenstein, C. Bertrán, R. Schlatter \& J. Tapia. 2006. Determinación del nivel de antropización de humedales como criterio para la planificación ecológica de la cuenca del Budi, Chile. Revista de Geografía Norte Grande 36: 75-91.

Peña-Cortés, F., J. Pincheira-Ulbrich, C. Bertrán, J. Tapia, E. Hauenstein, E. Fernández \& D. Rozas. 2011. A study of the geographic distribution of swamp forest in the coastal zone of the Araucanía Region, Chile. Applied Geography 31(2): 545-555.

Ramírez, C., L. Steubing \& M. Alberdi. 1976. La familia Hymenophyllaceae (Pteridophyta) en el fundo San Martín, Valdivia, Chile. I. Taxonomía y Ecología. Medio Ambiente 3(2): 3-13.

Ramírez, C., F. Ferriere \& H. Figueroa. 1983. Estudio fitosociológico de los bosques pantanosos templados del sur de Chile. Revista Chilena de Historia Natural 56: 1126.

Ramírez, C., C. San Martín \& J. San Martín. 1995. Estructura florística de los bosques pantanosos de Chile Sur-Central. En: J.J. Armesto, C. Villagrán \& M.K. Arroyo (eds.), Ecología de los bosques nativos de Chile, pp. 215-234. Editorial Universitaria. Santiago, Chile.

Ramírez, C., V. Sandoval, C. San Martín, M. Álvarez, Y. Pérez \& 
C. NovoA. 2012. El paisaje rural antropogénico de Aisén, Chile: Estructura y dinámica de la vegetación. Gayana Botanica 69(2): 219-231.

RaunKiaer, C. 1937. Plant life forms. Claredon, Oxford. 104 pp. Rodríguez, R., E. Ruiz \& J.P. Elissetche. 2005. Árboles en Chile. Editorial Universidad de Concepción, Chile. 212 pp.

Rodríguez, R., D. Alarcón \& J. Espejo. 2009. Helechos nativos del centro y sur de Chile. Guía de Campo. Corporación Chilena de la Madera (CORMA). Concepción, Chile. 212 pp.

San Martín, C., C. Ramírez \& J. San Martín. 2008. Distribución geográfica de los bosques pantanosos de Mirtáceas en Chile. Revista Geográfica de Chile Terra Australis 51/52: 49-64.

San Martín, J., A. Troncoso, C. Ramírez, C. San Martín \& A. Duarte. 1990. Estudio florístico y vegetacional de los bosques pantanosos nativos de la cordillera costera entre los ríos Rapel y Mataquito, Chile central. Revista Geográfica de Chile, Terra Australis 33: 103-128.

San Martín, J., A. Espinosa, S. Zanetti, E. Hauenstein, N. Ojeda \& C. Arriagada. 2008. Composición y estructura de la vegetación epífita vascular en un bosque primario de Olivillo (Aextoxicon punctatum R. et P.) en el sur de Chile. Ecología Austral 18: 1-11.

Steubing, L., R. Godoy \& M. Alberdi. 2002. Métodos de ecología vegetal. Editorial Universitaria,Santiago, Chile. 345 pp.

Weinberger, P., M. Romero \& M. Oliva. 1973. Investigaciones sobre la resistencia a la sequía de vegetales leñosos siempreverdes de la Patagonia. Vegetatio 28: 75-98.

Zuloaga, F., O. Morrone \& M. Belgrano. 2008. Catálogo de las plantas vasculares del Cono Sur (Argentina, Sur de Brasil, Chile, Paraguay y Uruguay). Missouri Botanical Garden Press, Saint Louis, USA. 3348 pp.

Anexo I. Catálogo general de la flora componente de los bosques pantanosos del borde costero de la Región de La Araucanía, Chile. (FV = forma de vida, $\mathrm{OF}=$ origen fitogeográfico, $\mathrm{EC}=$ estado de conservación; $\mathrm{E}=$ epífito, $\mathrm{L}=$ liana/trepadora, $\mathrm{P}=$ parásito, $\mathrm{C}=$ caméfito, $\mathrm{Cr}=$ criptófito, $\mathrm{Hc}=$ hemicriptófito, $\mathrm{F}=$ fanerófito, $\mathrm{Nf}=$ nanofanerófito, $\mathrm{Te}=$ terófito; $\mathrm{N}=$ nativo, $\mathrm{En}=$ endémico, $\mathrm{I}=$ introducido).

AnNex I. Catalogue of the flora of the swampy forests of the coastal edge of the La Araucanía Region, $\mathrm{Chile}(\mathrm{FV}=$ life form, $\mathrm{OF}=$ phytogeographycal origin, $\mathrm{EC}=$ conservation state, $\mathrm{E}=$ epiphyte, $\mathrm{L}=$ vine, $\mathrm{P}=$ parasite, $\mathrm{C}=$ chamaephyte, $\mathrm{Cr}=$ cryptophyte, $\mathrm{Hc}=$ hemicryptophyte, $\mathrm{F}=$ phanerophyte, $\mathrm{Nf}=$ nanophanerophyte, $\mathrm{Te}=$ therophyte, $\mathrm{N}=$ native, En= endemic, $\mathrm{I}=$ introduced).

\begin{tabular}{|c|c|c|c|c|}
\hline CLASE / Nombre Científico & FAMILIA & Nombre Común & FV & $\mathrm{OF}$ \\
\hline \multicolumn{5}{|l|}{ SPHENOPSIDA } \\
\hline Equisetum bogotense Kunth & Equisetaceae & Limpia plata & $\mathrm{Cr}$ & $\mathrm{N}$ \\
\hline \multicolumn{5}{|l|}{ FILICOPSIDA } \\
\hline Asplenium dareoides A.N. Desv. & Aspleniaceae & Filu-lahuén & $\mathrm{E}$ & $\mathrm{N}$ \\
\hline Asplenium trilobum Cav. & Aspleniaceae & s.n. & $\mathrm{E}$ & $\mathrm{N}$ \\
\hline Blechnum chilense (Kaulf.) Mett. & Blechnaceae & Costilla de vaca & $\mathrm{Cr}$ & $\mathrm{N}$ \\
\hline Blechnum hastatum Kaulf. & Blechnaceae & Palmilla & $\mathrm{Hc}$ & $\mathrm{N}$ \\
\hline Blechnum mochaenum Kunkel & Blechnaceae & Iquide & $\mathrm{Hc}$ & $\mathrm{N}$ \\
\hline Blechnum penna-marina (Poir.) Kuhn & Blechnaceae & Punke & $\mathrm{Hc}$ & En \\
\hline Grammitis magellanica Desv. & Grammitidaceae & s.n. & E & $\mathrm{N}$ \\
\hline Hymenoglossum cruentum (Cav.) K. Presl & Hymenophyllaceae & Helecho película & $\mathrm{E}$ & En \\
\hline Hymenophyllum caudiculatum Mart. var. productum (K. Presl) C. Chr. & Hymenophyllaceae & Pallante chilote & $\mathrm{E}$ & En \\
\hline Hymenophyllum dentatum Cav. & Hymenophyllaceae & Shushu-lahuén & $\mathrm{E}$ & $\mathrm{N}$ \\
\hline Hymenophyllum dicranotrichum (K.Presl) Hook. ex Sadeb. & Hymenophyllaceae & Helecho película & $\mathrm{E}$ & En \\
\hline Hymenophyllum krauseanum Phil. & Hymenophyllaceae & Helecho película & $\mathrm{E}$ & $\mathrm{N}$ \\
\hline Hymenophyllum pectinatum Cav. & Hymenophyllaceae & Helecho película & $\mathrm{E}$ & $\mathrm{N}$ \\
\hline Hymenophyllum peltatum (Poir.) Desv. & Hymenophyllaceae & Helecho película & $\mathrm{E}$ & $\mathrm{N}$ \\
\hline Hymenophyllum plicatum Kaulf. & Hymenophyllaceae & Helecho película & $\mathrm{E}$ & $\mathrm{N}$ \\
\hline Hymenophyllum tortuosum Hook. \& Grev. & Hymenophyllaceae & Helecho película & E & $\mathrm{N}$ \\
\hline Hypolepis poeppigii (Kunze) R.A. Rodr. & Dennstaedtiaceae & Huilel-lahuén & $\mathrm{Hc}$ & $\mathrm{N}$ \\
\hline Synammia feuillei (Bertero) Copel. var. feuillei & Polypodiaceae & Calahuala & $\mathrm{E}$ & $\mathrm{N}$ \\
\hline \multicolumn{5}{|l|}{ MAGNOLIOPSIDA (Dicotyledoneae) } \\
\hline Amomyrtus luma (Molina) D. Legrand \& Kausel & Myrtaceae & Luma & $\mathrm{F}$ & $\mathrm{N}$ \\
\hline Amomyrtus meli (Phil.) D. Legrand \& Kausel & Myrtaceae & Meli & $\mathrm{F}$ & En \\
\hline
\end{tabular}




\begin{tabular}{|c|c|c|c|c|}
\hline CLASE / Nombre CientíFico & FAMILIA & Nombre Común & $\mathrm{FV}$ & $\mathrm{OF}$ \\
\hline Antidaphne punctulata (Clos) Kuijt & Eremolepidaceae & Quintral & $\mathrm{P}$ & En \\
\hline Aristotelia chilensis (Molina) Stuntz & Elaeocarpaceae & Maqui & $\mathrm{F}$ & $\mathrm{N}$ \\
\hline Azara serrata Ruiz \& Pav. & Flacourtiaceae & Aromo & $\mathrm{Nf}$ & En \\
\hline Baccharis sagittalis (Less.) DC. & Asteraceae & Verbena 3 esquina & $\mathrm{C}$ & $\mathrm{N}$ \\
\hline Baccharis sphaerocephala Hook. \& Arn. & Asteraceae & Rari & $\mathrm{Nf}$ & En \\
\hline Berberis darwinii Hook. & Berberidaceae & Michay & $\mathrm{Nf}$ & $\mathrm{N}$ \\
\hline Berberis microphylla $\mathrm{G}$. Forst. & Berberidaceae & Calafate & $\mathrm{Nf}$ & $\mathrm{N}$ \\
\hline Berberis trigona Kunze ex. Poepp. et Endl. & Berberidaceae & Calafate, Michay & $\mathrm{Nf}$ & $\mathrm{N}$ \\
\hline Blepharocalyx cruckshanksii (Hook. \& Arn.) Nied. & Myrtaceae & Temu & $\mathrm{F}$ & En \\
\hline Boquila trifoliolata (DC.) Decne. & Lardizabalaceae & Pilpil-voqui & $\mathrm{L}$ & $\mathrm{N}$ \\
\hline Callitriche lechleri (Hegelm.) Fassett & Callitrichaceae & Huenchecó & $\mathrm{Cr}$ & I \\
\hline Centella asiatica (L.) Urb. & Apiaceae & Centella & $\mathrm{Hc}$ & $\mathrm{N}$ \\
\hline Cissus striata Ruiz \& Pav. & Vitaceae & Pilpil-voqui & $\mathrm{L}$ & $\mathrm{N}$ \\
\hline Dichondra sericea $\mathrm{Sw}$. & Convolvulaceae & Oreja de ratón & $\mathrm{Hc}$ & $\mathrm{N}$ \\
\hline Drimys winteri J.R. Forst. \& G. Forst. & Winteraceae & Canelo & $\mathrm{F}$ & $\mathrm{N}$ \\
\hline Ercilla syncarpellata Nowicke & Phytolaccaceae & Voqui traro & $\mathrm{L}$ & En \\
\hline Escallonia revoluta (Ruiz \& Pav.) Pers. & Escalloniaceae & 7 camisas & $\mathrm{Nf}$ & En \\
\hline Fuchsia magellanica Lam. & Onagraceae & Chilco & $\mathrm{Nf}$ & $\mathrm{N}$ \\
\hline Galium tricornutum Dandy & Rubiaceae & s.n. & $\mathrm{Te}$ & I \\
\hline Gratiola peruviana $\mathrm{L}$. & Plantaginaceae & Contrahierba & $\mathrm{Cr}$ & $\mathrm{N}$ \\
\hline Gunnera tinctoria (Molina) Mirb. & Gunneraceae & Nalca & $\mathrm{Cr}$ & $\mathrm{N}$ \\
\hline Hydrocotyle chamaemorus Cham. \& Schltdl. & Apiaceae & Sombrero de agua & $\mathrm{Hc}$ & $\mathrm{N}$ \\
\hline Hydrocotyle modesta Cham. \& Schltdl. & Apiaceae & Sombrero de agua & $\mathrm{Hc}$ & $\mathrm{N}$ \\
\hline Hydrocotyle ranunculoides L.f. & Apiaceae & Sombrero de agua & $\mathrm{Hc}$ & $\mathrm{N}$ \\
\hline Lepidoceras chilense (Molina) Kuijt & Eremolepidaceae & Quintral del temu & $\mathrm{P}$ & En \\
\hline Leptinella scariosa Cass. & Asteraceae & Botón de oro & $\mathrm{Hc}$ & $\mathrm{N}$ \\
\hline Lomatia ferruginea (Cav.) R. Br. & Proteaceae & Fuinque & $\mathrm{F}$ & $\mathrm{N}$ \\
\hline Lomatia hirsuta (Lam.) Diels ex J.F. Macbr. & Proteaceae & Radal & $\mathrm{F}$ & $\mathrm{N}$ \\
\hline Lotus pedunculatus Cav. & Fabaceae & Alfalfa chilota & $\mathrm{Hc}$ & I \\
\hline Luma apiculata (DC.) Burret & Myrtaceae & Arrayán & $\mathrm{F}$ & $\mathrm{N}$ \\
\hline Luma chequen (Molina) A. Gray & Myrtaceae & Chin-chin & $\mathrm{F}$ & En \\
\hline Maytenus boaria Molina & Celastraceae & Maitén & $\mathrm{F}$ & $\mathrm{N}$ \\
\hline Mitraria coccinea Cav. & Gesneriaceae & Botellita & $\mathrm{L}$ & $\mathrm{N}$ \\
\hline Muehlenbeckia hastulata (Sm.) I.M.Johnst. & Polygonaceae & Quilo & $\mathrm{L}$ & $\mathrm{N}$ \\
\hline Myoschilos oblongum Ruiz \& Pav. & Santalaceae & Orocoipo & $\mathrm{Nf}$ & $\mathrm{N}$ \\
\hline Myosotis scorpioides L. & Boraginaceae & No me olvides & $\mathrm{Hc}$ & $\mathrm{I}$ \\
\hline Myrceugenia chrysocarpa (O.Berg) Kausel & Myrtaceae & Luma blanca & $\mathrm{F}$ & $\mathrm{N}$ \\
\hline Myrceugenia exsucca (DC.) O.Berg & Myrtaceae & Pitra & $\mathrm{F}$ & $\mathrm{N}$ \\
\hline Myrceugenia planipes (Hook.\& Arn.) O.Berg & Myrtaceae & Picha-picha & $\mathrm{F}$ & $\mathrm{N}$ \\
\hline Nasturtium officinale R.Br. & Brassicaceae & Berro & $\mathrm{Cr}$ & I \\
\hline Nertera granadensis (Mutis ex L.f.) Druce & Rubiaceae & Coralillo & $\mathrm{Hc}$ & $\mathrm{N}$ \\
\hline Notanthera heterophylla (Ruiz\& Pav.) G. Don & Loranthaceae & Quintral del boldo & $\mathrm{P}$ & En \\
\hline Oldenlandia salzmannii (DC.) Benth. \& Hook. & Rubiaceae & s.n. & $\mathrm{Hc}$ & $\mathrm{N}$ \\
\hline Pilea elliptica Hook. f. & Urticaceae & Pilea & $\mathrm{Hc}$ & En \\
\hline
\end{tabular}




\begin{tabular}{|c|c|c|c|c|}
\hline CLASE / Nombre Científico & FAMILIA & Nombre Común & $\mathrm{FV}$ & $\mathrm{OF}$ \\
\hline Polygonum hydropiperoides Michx. & Polygonaceae & Duraznillo & $\mathrm{Hc}$ & $\mathrm{N}$ \\
\hline Prunella vulgaris $\mathrm{L}$. & Lamiaceae & Hierba mora & $\mathrm{C}$ & I \\
\hline Ranunculus repens $\mathrm{L}$. & Ranunculaceae & Botón de oro & $\mathrm{Hc}$ & $\mathrm{I}$ \\
\hline Raukaua valdiviensis (Gay) Frodin & Araliaceae & Voqui naranjillo & $\mathrm{L}$ & En \\
\hline Rhamnus diffusus Clos & Rhamnaceae & Murta negra & $\mathrm{Nf}$ & En \\
\hline Ribes trilobum Meyen & Grossulariaceae & Zarzaparrilla & $\mathrm{Nf}$ & En \\
\hline Rubus constrictus P.J. Müll. \& Léfebre & Rosaceae & Zarzamora & $\mathrm{Nf}$ & $\mathrm{I}$ \\
\hline Salix babylonica L. & Salicaceae & Sauce llorón & $\mathrm{F}$ & I \\
\hline Salix caprea L. & Salicaceae & Sauce capruno & $\mathrm{F}$ & $\mathrm{I}$ \\
\hline Salix viminalis $\mathrm{L}$. & Salicaceae & Sauce mimbre & $\mathrm{F}$ & $\mathrm{I}$ \\
\hline Sarmienta scandens (J.D. Brandis ex Molina) Pers. & Gesneriaceae & Medallita & $\mathrm{E}$ & En \\
\hline Senecio fistulosus Poepp. ex Less. & Asteraceae & Hualtata & $\mathrm{Hc}$ & $\mathrm{N}$ \\
\hline Tepualia stipularis (Hook.\& Arn.) Griseb. & Myrtaceae & Tepú & $\mathrm{F}$ & $\mathrm{N}$ \\
\hline Tristerix corymbosus (L.) Kuijt & Loranthaceae & Quintral & $\mathrm{P}$ & $\mathrm{N}$ \\
\hline \multicolumn{5}{|l|}{ LILIOPSIDA (Monocotyledoneae) } \\
\hline Agrotis capillaris L. & Poaceae & Chépica & $\mathrm{Hc}$ & I \\
\hline Carex acutata Boott & Cyperaceae & Cortadera & $\mathrm{Hc}$ & En \\
\hline Chusquea quila Kunth & Poaceae & Quila & $\mathrm{Cr}$ & En \\
\hline Chusquea uliginosa Phil. & Poaceae & Quila & $\mathrm{Cr}$ & En \\
\hline Cyperus eragrostis Lam. & Cyperaceae & Cortadera & $\mathrm{Hc}$ & $\mathrm{N}$ \\
\hline Eleocharis acicularis (L.) Roem.\& Schult. & Cyperaceae & Rume & $\mathrm{Cr}$ & $\mathrm{N}$ \\
\hline Fascicularia bicolor (Ruiz \& Pav.) Mez & Bromeliaceae & Chupalla & $\mathrm{E}$ & En \\
\hline Greigia sphacelata Regel & Bromeliaceae & Chupón & $\mathrm{Hc}$ & En \\
\hline Isolepis cernua (Vahl) Roem. \&Schult. & Cyperaceae & s.n. & $\mathrm{Te}$ & $\mathrm{N}$ \\
\hline Juncus procerus E. Meyer & Juncaceae & Junquillo & $\mathrm{Hc}$ & $\mathrm{N}$ \\
\hline Lapageria rosea Ruiz \& Pav. & Philesiaceae & Copihue & $\mathrm{L}$ & En \\
\hline Luzuriaga polyphylla (Hook.) J.F. Macbr. & Luzuriagaceae & Coralillo, quilineja & $\mathrm{L}$ & En \\
\hline Luzuriaga radicans Ruiz \& Pav. & Luzuriagaceae & Coralillo, quilineja & $\mathrm{L}$ & $\mathrm{N}$ \\
\hline Scirpus inundatus (R.Br.) Poir. & Cyperaceae & Can Can & $\mathrm{Cr}$ & $\mathrm{N}$ \\
\hline Triglochin palustris L. & Juncaginaceae & Hierba paloma & $\mathrm{Cr}$ & $\mathrm{N}$ \\
\hline
\end{tabular}

AneXo 2. Distribución de las especies en los rodales de las seis cuencas estudiadas ( $+=$ presencia, - = ausencia).

AnNex 2. Distribution of the species in the stands of the six basins studied ( $+=$ presence, $-=$ absence).

\begin{tabular}{|c|c|c|c|c|c|c|}
\hline ESPECIES & IMPERIAL & CoMúE & Chelle & TOLTÉN & BOROA & BOLDO \\
\hline Agrotis capillaris & + & - & - & - & - & + \\
\hline Amomyrtus luma & - & + & + & + & + & + \\
\hline Amomyrtus meli & + & - & + & + & - & + \\
\hline Antidaphne punctulata & - & - & + & - & + & - \\
\hline Aristotelia chilensis & + & - & + & - & - & + \\
\hline Asplenium dareoides & - & - & + & + & + & - \\
\hline Asplenium trilobum & - & - & + & + & - & - \\
\hline Azara serrata & - & - & - & + & - & + \\
\hline Baccharis sagittalis & - & - & + & - & - & - \\
\hline
\end{tabular}


Gayana Bot. 71(1), 2014

\begin{tabular}{|c|c|c|c|c|c|c|}
\hline ESPECIES & IMPERIAL & ComúE & Chelle & TOLTÉN & BOROA & Boldo \\
\hline Baccharis sphaerocephala & - & - & - & - & + & + \\
\hline Berberis darwinii & - & + & - & - & - & - \\
\hline Berberis microphylla & - & + & + & - & - & - \\
\hline Berberis trigona & - & - & - & + & - & - \\
\hline Blechnum chilense & + & + & + & + & + & + \\
\hline Blechnum hastatum & - & - & - & - & - & + \\
\hline Blechnum mochaenum & - & - & - & - & - & + \\
\hline Blechnum penna-marina & - & - & - & - & - & + \\
\hline Blepharocalyx cruckshanksii & + & + & + & + & + & + \\
\hline Boquila trifoliolata & + & + & + & + & + & + \\
\hline Callitriche lechleri & - & - & - & - & - & + \\
\hline Carex acutata & - & - & - & - & - & + \\
\hline Centella asiatica & - & - & - & + & - & + \\
\hline Chusquea quila & - & - & - & + & - & + \\
\hline Chusquea uliginosa & - & - & - & + & + & + \\
\hline Cissus striata & + & + & + & + & + & + \\
\hline Cyperus eragrostis & + & + & + & + & + & + \\
\hline Dichondra sericea & - & - & + & - & - & - \\
\hline Drimys winteri & + & + & + & + & + & + \\
\hline Eleocharis acicularis & + & - & - & + & - & + \\
\hline Equisetum bogotense & - & - & - & + & - & - \\
\hline Ercilla syncarpellata & - & - & - & + & - & + \\
\hline Escallonia revoluta & + & - & + & - & + & - \\
\hline Fascicularia bicolor & - & + & + & + & + & + \\
\hline Fuchsia magellanica & + & + & - & + & + & + \\
\hline Galium tricornutum & - & - & - & + & - & - \\
\hline Grammitis magellanica & - & - & - & + & - & + \\
\hline Gratiola peruviana & - & - & - & + & - & + \\
\hline Greigia sphacelata & - & - & - & - & - & + \\
\hline Gunnera tinctoria & - & - & - & + & - & - \\
\hline Hydrocotyle chamaemorus & - & - & - & + & - & - \\
\hline Hydrocotyle modesta & - & + & + & - & - & + \\
\hline Hydrocotyle ranunculoides & - & - & + & - & - & - \\
\hline Hymenoglossum cruentum & - & - & + & - & - & - \\
\hline Hymenophyllum caudiculatum & - & - & + & - & - & - \\
\hline H. dentatum & - & - & + & - & - & + \\
\hline H. dicranotrichum & - & - & + & - & - & + \\
\hline H. krauseanum & - & - & + & + & + & + \\
\hline H. pectinatum & - & - & - & + & - & - \\
\hline H. peltatum & - & - & + & - & - & + \\
\hline H. plicatum & - & - & + & - & - & + \\
\hline H. tortuosum & - & - & - & - & - & + \\
\hline Hypolepis poeppigii & - & - & - & - & + & - \\
\hline Isolepis cernua & - & - & + & - & - & - \\
\hline Juncus procerus & - & + & + & + & + & + \\
\hline Lapageria rosea & - & - & + & + & - & - \\
\hline
\end{tabular}


Florística y degradación del bosque pantanoso: HaUENSTEIN, E. ET AL.

\begin{tabular}{|c|c|c|c|c|c|c|}
\hline EsPeCIES & IMPERIAL & ComúE & Chelle & TOLTÉN & BOROA & BOLDO \\
\hline Lepidoceras chilense & - & + & + & + & + & + \\
\hline Leptinella scariosa & - & - & - & + & - & - \\
\hline Lomatia ferruginea & - & + & - & + & - & + \\
\hline Lomatia hirsuta & - & - & - & - & - & + \\
\hline Lotus pedunculatus & - & + & + & - & - & + \\
\hline Luma apiculata & - & - & + & + & - & + \\
\hline Luma chequen & + & - & + & + & - & + \\
\hline Luzuriaga polyphylla & + & - & - & + & + & + \\
\hline Luzuriaga radicans & - & - & + & + & + & + \\
\hline Maytenus boaria & - & + & + & + & - & - \\
\hline Mitraria coccinea & - & - & + & + & + & + \\
\hline Muehlenbeckia hastulata & + & + & + & + & + & + \\
\hline Myoschilos oblongum & - & - & - & - & - & + \\
\hline Myosotis scorpioides & - & - & - & + & - & + \\
\hline Myrceugenia chrysocarpa & - & - & + & - & + & + \\
\hline Myrceugenia exsucca & + & + & + & + & + & + \\
\hline Myrceugenia planipes & - & - & + & - & + & - \\
\hline Nasturtium officinale & - & - & - & - & + & + \\
\hline Nertera granadensis & + & - & - & + & - & + \\
\hline Notanthera heterophylla & - & - & - & - & - & + \\
\hline Oldenlandia salzmannii & - & - & - & + & - & - \\
\hline Pilea elliptica & - & - & - & + & + & + \\
\hline Polygonum hydropiperoides & + & - & + & + & - & + \\
\hline Prunella vulgaris & - & + & + & + & - & - \\
\hline Ranunculus repens & + & - & - & + & - & + \\
\hline Raukaua valdiviensis & - & - & + & - & - & + \\
\hline Rhamnus diffusus & + & - & + & - & - & - \\
\hline Ribes trilobum & + & + & + & + & - & + \\
\hline Rubus constrictus & + & + & + & + & + & + \\
\hline Salix babylonica & + & - & - & - & - & - \\
\hline Salix caprea & - & + & + & + & - & - \\
\hline Salix viminalis & - & - & - & + & - & - \\
\hline Sarmienta scandens & - & + & + & + & + & + \\
\hline Scirpus inundatus & - & - & - & - & - & + \\
\hline Senecio fistulosus & - & - & + & - & - & - \\
\hline Synammia feuillei & - & - & + & + & + & + \\
\hline Tepualia stipularis & - & - & - & - & - & + \\
\hline Triglochin palustris & - & - & - & + & - & - \\
\hline Tristerix corymbosus & - & - & + & + & - & + \\
\hline Total especies (94) & 23 & 24 & 51 & 55 & 30 & 61 \\
\hline
\end{tabular}

Recibido: 08.03.13

Aceptado: 16.10.13 